\title{
Filling and shielding for postoperative gastric perforations of endoscopic submucosal dissection using polyglycolic acid sheets and fibrin glue
}

Authors

Institutions
Kengo Takimoto ${ }^{1}$, Akeo Hagiwara ${ }^{2}$

Takeda General Hospital, Department of Gastroenterology, Kyoto, Japan

${ }^{2}$ Doshisya University, Faculty of Life and Medical Sciences, Kyoto, Japan submitted

3. November 2014 accepted after revision 21. March 2016

\section{Bibliography}

DOI http://dx.doi.org/ 10.1055/s-0042-105867 Endoscopy International Open 2016; 04: E661-E664

(c) Georg Thieme Verlag KG Stuttgart · New York E-ISSN 2196-9736

\section{Corresponding author} Kengo Takimoto, MD, PhD Department of

Gastroenterology Takeda General Hospital 28-1 Ishidamori minamimachi Fushimi-ku Kyoto 601-1495

Japan

k-takimoto-kengo-12091209@y8.dion.ne.jp
Background and study aims: Many medical institutions in Japan perform endoscopic mucosal dissection (ESD) to treat early gastric cancer. Perforations can occur during ESD, and clipping has been reported as useful for treating small pinhole perforations. However, it is often difficult to close postoperative perforations because they usually have large diameters, and the muscle layer around the perforated region is often fragile, so additional open surgery is the only currently used method to treat large perforations and delayed perforations. Another method for large per-

\section{Introduction

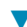

Endoscopic submucosal dissection (ESD) has become standard treatment for early gastric cancer but if postoperative perforation occurs, surgical intervention is required [1]. Clip closure has been reported to be useful for closing intraoperative perforations [2], but it is often difficult to close postoperative perforations because they usually have large diameters, and the muscle layer around the perforated region is often fragile. Even if clip closure is applicable, we have encountered cases in which the clip subsequently fell off, causing the perforation to recur.

Ono et al. reported a case in which a postoperative perforation was closed using a polyglycolic acid (PGA) sheet and fibrin glue [3]. This method is currently used to occlude postoperative pulmonary fistulas [4]. In addition, it has been used by the authors' group to repair duodenal injuries that occur during ESD. We report 3 cases in which PGA sheets and fibrin glue were successfully used to repair postoperative gastric perforations endoscopically. Moreover, we were able to view the healing of the postoperative perforations by using a shielding method. foration is needed to treat perforations endoscopically. Ono et al. reported a case in which a postoperative perforation was closed using a polyglycolic acid (PGA) sheet and fibrin glue. In addition, it has been used by the authors' group to repair duodenal injuries that occur during ESD. We report 3 cases in which PGA sheets and fibrin glue were successfully used to repair postoperative gastric perforations endoscopically. This method is simple, safe, and effective, and is a new way to treat large perforations and delayed perforations that occur following ESD.

\section{Case Reports \\ $\nabla$}

Of 1150 patients who underwent gastric ESD at our hospital between 2004 and 2013, postoperative perforation occurred in 5 . We repaired the perforations using PGA sheets (Neoveil ${ }^{\circledR} 015$, Gunze Limited, Japan) and fibrin glue (Bolheal ${ }^{\circledR}$, Kaketsuken, Japan, or Beriplast ${ }^{\circledR}$ P, CSL Berling, USA) in 3 of these patients ( Table 1 ). Application of the current method had been approved by the ethics committee for all possible cases in advance. Thus, we were able to obtain the informed consent from the patient before each application of this method without delay.

All 3 patients developed symptoms of abdominal pain and fever on the day of the ESD. In all cases, computed tomography (CT) showed free air in the intraperitoneal cavity. Emergency upper gastrointestinal endoscopy was performed, which revealed extensive separation of the muscular layers around the perforated region in all cases. Clipping was attempted, but the perforations were large and could not be occluded.

\section{Patient 1}

The patient was a 74-year-old man with earlystage gastric cancer (clinical type 0-IIa, diameter $20 \mathrm{~mm}$ ) in the greater curvature of the gastric fornix. The tumor was removed by ESD. During sur- 
Table 1 Clinicopathological features and clinical outcomes of 3 patients treated with the shielding method.

\begin{tabular}{|c|c|c|c|c|c|c|c|c|c|c|}
\hline $\begin{array}{l}\text { Case } \\
\text { no. }\end{array}$ & $\begin{array}{l}\text { Age } \\
\text { (years) }\end{array}$ & Sex & Multiple tumors & $\begin{array}{l}\text { Tumor } \\
\text { location }\end{array}$ & $\begin{array}{l}\text { Tumor } \\
\text { size } \\
(\mathrm{mm})\end{array}$ & $\begin{array}{l}\text { Depth of } \\
\text { tumor }\end{array}$ & $\begin{array}{l}\text { Scar in } \\
\text { tumor }\end{array}$ & Histological type & $\begin{array}{l}\text { Resected } \\
\text { specimen } \\
\text { size }(\mathrm{mm})\end{array}$ & $\begin{array}{l}\text { Time to post- } \\
\text { operative per- } \\
\text { foration (h) }\end{array}$ \\
\hline 1 & 74 & M & Post-gastric cancer surgery & Fornix & 20 & $\mathrm{~m}$ & - & differentiated & 30 & 6 \\
\hline 2 & 85 & $\mathrm{~F}$ & No & Body & 40 & $\mathrm{~m}$ & - & undifferentiated & 50 & 8 \\
\hline 3 & 79 & $\mathrm{~F}$ & No & Body & 15 & $\mathrm{~m}$ & - & differentiated & 30 & 9 \\
\hline
\end{tabular}

gery, no perforation was observed. However, 6 hours after the procedure, the patient suddenly developed severe abdominal pain. After $\mathrm{CT}$, emergency endoscopy was performed under $\mathrm{CO}_{2}$ insufflation. Endoscopy revealed a post-ESD ulcer and a postoperative gastric perforation measuring $20 \mathrm{~mm}$ involving extensive separation of all muscle layers ( $\bullet$ Fig. 1 a). Because an attempt at clipping failed, the endoscopic filling and shielding method described here was used. The patient was discharged from the hospital after 20 days.

\section{Patient 2}

The patient was an 85-year-old woman who presented with early-stage gastric cancer (clinical type 0 -IIc, diameter $40 \mathrm{~mm}$ ) in the posterior wall of the gastric body. The tumor was removed by ESD. During surgery, no perforation was observed, but the patient developed abdominal pain 8 hours after the procedure. Upper gastrointestinal endoscopy revealed a post-ESD ulcer and a postoperative gastric perforation (size $30 \mathrm{~mm}$ ) involving extensive separation of all muscle layers of the greater curvature of the stomach. Because clipping failed, endoscopic filling and shielding were performed. The patient was hospitalized for 28 days.

\section{Patient 3}

The patient was a 79-year-old woman with early-stage gastric cancer (clinical type 0-IIa, diameter $15 \mathrm{~mm}$ ) in the posterior wall of the gastric body. The tumor was removed by ESD. No perforation was observed during the ESD. However, the patient developed abdominal pain 9 hours after the procedure. The following day, upper gastrointestinal endoscopy was performed, which revealed a post-ESD ulcer and a postoperative gastric perforation measuring slightly less than $10 \mathrm{~mm}$. Because we judged that the clip might have become detached, endoscopic filling and shielding were performed. The patient was hospitalized for 12 days.

In each case, the procedure was performed in accordance with our previously described method [5].

Step 1 . If the perforation was large, we first inserted a large sheet (measuring about $20 \times 20 \mathrm{~mm}$ ) into the cavity and placed it over the perforation site to make a bed-like space for building up the PGA sheets.

Step 2. A PGA sheet $(0.15 \mathrm{~mm}$ thick $)$ was cut into $15 \mathrm{~mm} \times 7 \mathrm{~mm}$ to $20 \times 20 \mathrm{~mm}$-sized pieces, which were then placed over the perforated site with biopsy forceps (FB-230, Olympus).

Step 3. The cut pieces were packed into the perforation, and a shield was inserted to cover the filled section and fixed in place with fibrin glue.

Step 4. Fibrin glue was applied using a spray tube for endoscopy. We advanced the spray tube into the PGA sheet-filled region so that solution A (fibrinogen) was a) inserted between the sheets and $b$ ) passed through them to the other side of the perforation. Step 5. Next, solution B (thrombin) was injected deeper inside the PGA sheet-filled region using another spray tube to form fibrin gel, which bonded the pieces of PGA sheet together (ه Fig. 1 b).

In our cases, upper gastrointestinal endoscopy was performed 2 days to 4 days after the procedure, and revealed that the perforations had been completely covered by the PGA sheets. The PGA sheets were still visible 1 week to 2 weeks later ( Fig. 1 c). Four weeks after the procedure, granulation tissue had completely covered the perforated sections in all cases, and the PGA sheets were no longer present. The duration of the procedure was 15 minutes on average, and there were no adverse events. During endoscopic examinations performed 7 weeks to 9 months after the procedure, it was found that the perforation site was scarred in all cases, and complete occlusion of the perforation without any intraperitoneal communication was confirmed ( $\mathbf{F i g} . \mathbf{1 d}$ ). None of the patients required additional surgery.

\section{Discussion}

$\nabla$

Several studies have reported that, in most cases of postoperative perforation, emergency surgical procedures are employed because large and fragile perforations cannot be closed. [1,3]

At our hospital, we encountered 2 other cases of postoperative perforation in which additional open surgery was required. The spontaneous healing of postoperative perforations might be very rare. First, we employed a method of clip closure. When clip closure could not be occluded, we employed a method involving endoscopic filling and shielding. We always consult with a surgeon and the patients who are candidates for this surgery. When the surgeon determines that a watchful-waiting strategy is appropriate, we follow up carefully without additional surgery. In addition, we confirm that a patient is not suffering from sepsis or pan-peritonitis before perform the PGA filling and shielding method.

Ono et al. reported a case in which PGA sheets were used [3]. In our cases, the perforations were much larger than that described in the latter report. Moreover, an examination of the perforation healing process revealed that granulation tissue formed below the PGA sheet, which was gradually pushed out of the perforated site.

Hanaoka et al. reported that emergency surgery was required in $83 \%$ ( 5 of $6,95 \%$ CI $54-100 \%$ ) of cases of postoperative perforation [1]. Emergency surgery is challenging and is associated with high risks in hospitals with limited resources. There are also risks associated with general anesthesia, and an omentum patch or subtotal gastrectomy might be required after postoperative perforation.

In general, clipping has been the only endoscopic method used to treat perforations. However, it can be extremely difficult or even impossible to close large perforations with clipping. Even if clip closure can be performed, the perforated section and the surrounding mucous membrane are often extremely fragile, and 


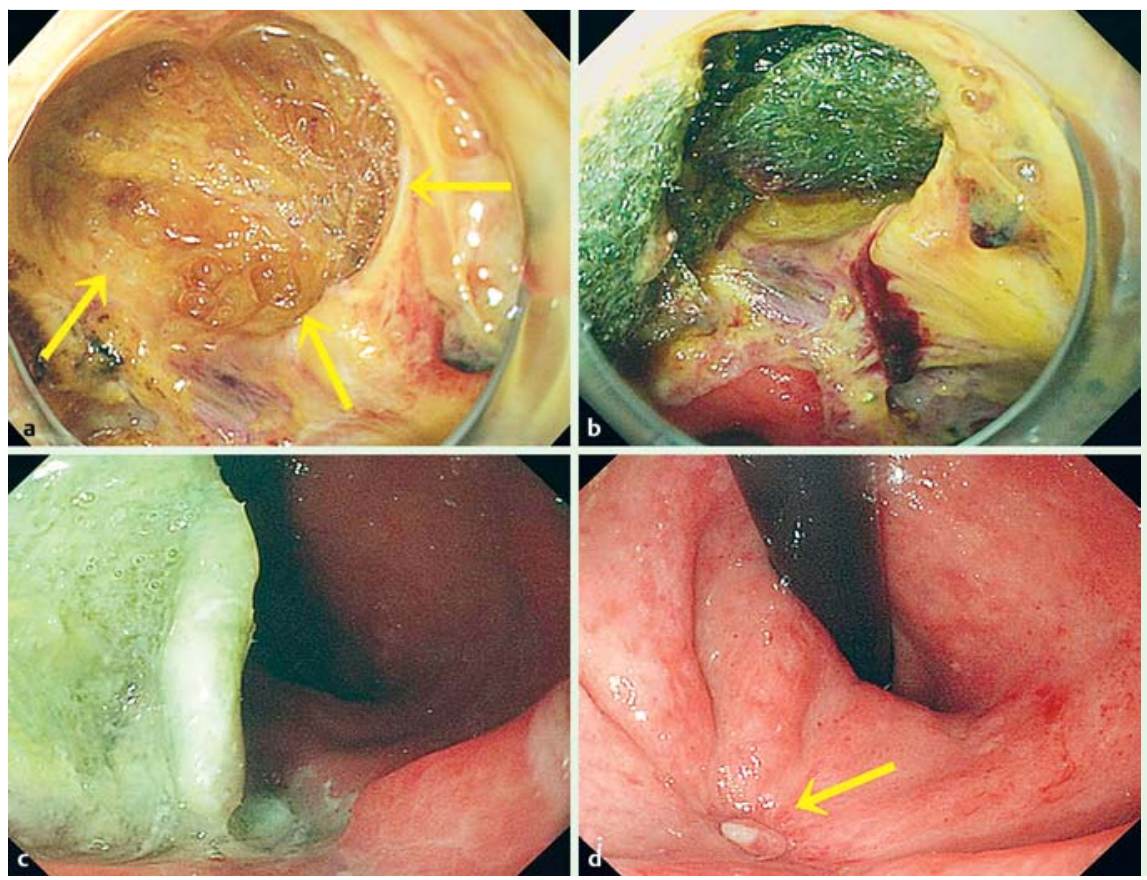

Fig. 1 Patient 1 of the case series. a A postoperative perforation about $20 \mathrm{~mm}$ occurred at POD 1 (yellow arrow). b Endoscopic tissue shielding with polyglycolic acid (PGA) sheets and fibrin glue for postoperative perforation. $\mathrm{c}$ Seven days after endoscopic mucosal dissection, the PGA sheets remained and covered the perforated site. $\mathbf{d}$ Ten weeks after the shielding method, the PGA sheets did not remain and the ulcer became scarred (yellow arrow).

the clips can easily become detached. Furthermore, in postoperative perforations, all muscle layers become separated. Even if the mucous membrane is closed, there is a high risk of re-perforation secondary to insufficient blood flow.

Clip closure using an Endoloop can close large gastrointestinal perforations [6], but clips are easily detached after postoperative perforation because the perforated site can be very fragile.

The Over-the-Scope Clip (OTSC) system might be useful for treating postoperative perforation of the gastrointestinal tract. However, Ono $\mathrm{H}$ et al. reported that special care is required when employing the OTSC in organs with a narrow lumen, such as the duodenum, because of the risk of stenosis. Furthermore, there are difficulties associated with using the OTSC for perforation closure. For example, the OTSC Twin Grasper can be awkward to manipulate, and treatment is sometimes difficult [7].

PGA sheets and fibrin glue are safe and have been used in the clinical setting in various surgical fields $[5,8]$. PGA sheets and fibrin glue are not recognized as foreign substances because they degrade in the human body within 4 weeks and 8 weeks, respectively, after application. In addition, they may have healing effects because they function as scaffolds for tissue regeneration [9]. Granulation tissue fills and covers perforations, generating scaffolds that can promote their closure. Indeed, granulation tissue formed in all 3 cases reported in the current study and none of the patients suffered reperforation, eliminating the need for surgery. In addition, follow-up endoscopy after the coating procedure detected residual PGA sheet material. The adhesive strength of the fibrin glue was strong enough to prevent the PGA sheets from detaching during gastrointestinal peristalsis. In the presence of perforation or inflammation, adhesion may be increased by the healing capacity of the human body. In the field of regenerative medicine, inflammation is considered to be essential for regeneration [10]. In the current study, inflammation might have contributed to the early formation of granulation tissue. In our hospital, we employed clip closure. We perform endoscopic filling and shielding for postoperative perforations when the following three criteria are met:
1. The patient is in good condition and free from pan-peritonitis and sepsis;

2. A surgeon has been consulted, and a watchful waiting strategy is employed; and

3. The diameter and depth of the perforated site make its covering and filling with PGA sheets feasible.

In cases in which a patient meets the above 3 requirements, we use the PGA filling and shielding method. After that, together with surgeons, we follow a patient carefully, and monitor his or her condition so as not to miss the window for further surgery, should a procedure be required.

We believe that patients who develop postoperative perforation and are still in good condition should have surgery. We always consulted with surgeons and patients who were candidates for those procedures. Fortunately, in 3 cases in which surgeons recommended a watchful-waiting strategy, the patients got better while being monitored. But we think this method is an exploratory treatment.

The method described here was performed in a single institution in a small number of subjects. Further cases should be accumulated to confirm the safety and efficacy of the filling and shielding method. At present, clipping closure is the only endoscopic method for closing postoperative perforations; therefore, when clipping closure fails, we propose that the method described in this study might be an alternative. In the current study, the shield did not become detached in any case. However, shield detachment is a possible complication that warrants further investigation.

The use of the method described here should be thoroughly explored because it may reduce the need for open surgery in patients with postoperative perforation. Endoscopic treatment using PGA sheets and fibrin glue might be of limited use for closing postoperative perforations involving very large and deep cavities.

\section{Competing interests: None}




\section{References}

1 Hanaoka $N$, Uedo $N$, Ishihara $R$ et al. Clinical features and outcomes of delayed perforation after endoscopic submucosal dissection for early gastric cancer. Endoscopy 2010; 42: 1112 - 1115

2 Minami S, Gotoda T, Ono H et al. Complete endoscopic closure of gastric perforation induced by endoscopic resection of early gastric cancer using endoclips can prevent surgery (with video). Gastrointest Endosc 2006; 63: 596-601

3 Ono H, Takizawa K, Kakushima $\mathrm{N}$ et al. Application of polyglycolic acid sheets for delayed perforation after endoscopic submucosal dissection of early gastric cancer. Endoscopy 2015; 47: E18-E19

$4 \mathrm{Kawai}$, Harada K, Ohta $\mathrm{H}$ et al. Prevention of alveolar air leakage after video-assisted thoracic surgery: comparison of the efficacy of methods involving the use of fibrin blue. Thorac Cardiovasc Surg 2012; 60: $351-355$

5 Takimoto K, Toyonaga T, Matsuyama $K$ et al. Endoscopic tissue shielding to prevent delayed perforation associated with endoscopic submu- cosal dissection for duodenal neoplasms. Endoscopy 2012; 44: 414 415

6 Abe S, Oda I, Mori G et al. Complete endoscopic closure of a large gastric defect with endoloop and endoclips after complex endoscopic submucosal dissection. Endoscopy 2015; 47: E374-E375

7 Ono H, Tanaka M, Takizawa K et al. Utility of the over-the-scope-clip system for treating a large esophageal perforation. Esophagus 2015; 12: $336-339$

8 Izumi Y, Kawamura M, Gika M et al. Granulation tissue formation at the bronchial stump is reduced after stapler closure in comparison to suture closure in dogs. Interact CardioVasc Thorac Surg 2010; 10: 356 359

9 Kroez M, Lang W, Dickneite G. Wound healing and degradation of the fibrin sealant Beriplast $P$ following partial liver resection in rabbits. Wound Repair Regen 2005; 13: 318 - 323

10 Gurtner GC, Werner S, Barrandon Y et al. Wound repair and regeneration. Nature 2008; 453: 314-321 